\title{
Octopamine Enhances Dark-Adaptation in Limulus Ventral Photoreceptors ${ }^{1}$
}

\author{
PETER M. O'DAY ${ }^{2}$ AND JOHN E. LISMAN ${ }^{3}$ \\ Department of Biology, Brandeis University, Waltham, Massachusetts 02254
}

\begin{abstract}
Limulus ventral photoreceptors receive synaptic input from fibers that emerge from the brain and that appear to use octopamine as a transmitter. Exogenous application of octopamine has been shown to increase levels of intracellular CAMP in ventral photoreceptors, but the resulting physiological effects have been unclear. In this report, we show that octopamine increases the rate of dark-adaptation following a bright light. Since a similar increase in the rate of darkadaptation is produced by IBMX (1 $\mathrm{mm}$ ) and forskolin (100 $\mu \mathrm{M})$, drugs shown previously to raise the concentration of CAMP, the octopamine effect may be mediated by cAMP. Our results suggest that dark-adaptation, a fundamental process of photoreceptors, is under efferent neural control.
\end{abstract}

The photoreceptors of Limulus ventral and lateral eyes receive synaptic input from efferent fibers originating in the brain (Fahrenbach, 1973). Octopamine is synthesized and stored in the efferent fibers (Evans et al., 1983) and released upon depolarization in a $\mathrm{Ca}^{2+}$-dependent manner (Battelle et al., 1982), indicating that octopamine is a synaptic transmitter at these synapses. There is also some evidence that substance $P$ may be contained in efferent fibers synapsing on the lateral eye, but the postsynaptic targets have not been clearly established (Chamberlain and Engbretson, 1982; Mancillas and Browil, 1984). In the ventral eye, however, no substance $P$ immunoreactivity has been detected (Chamberlain and Engbretson, 1982; Mancillas and Brown, 1984). Efferent spike activity to both ventral and lateral eyes undergoes circadian variation; activity is high during the night and low during the day (Barlow et al., 1977; Eisele et al., 1982).

In the lateral eye, several postsynaptic effects of this circadian input have been demonstrated (Chamberlain and Barlow, 1977; Barlow et al., 1980; Kaplan and Barlow, 1980; Barlow, 1983; Kass and Barlow, 1984). These include a reduction in the rate of occurrence of spontaneous quantum bumps and structural changes in the photoreceptors, changes that enhance the detection of dim lights. Recent evidence suggests that these postsynaptic effects may be mediated by cAMP (Kass and Barlow, 1982; Pelletier et al., 1984). In contrast, the role of the synaptic input into the ventral eye has been unclear. The sensitivity of the dark-adapted ventral eye to

Received June 26, 1984; Revised November 27, 1984;

Accepted November 28, 1984

\footnotetext{
${ }^{1}$ We wish to thank Drs. Barbara-Ann Battelle, Joel Brown, Steve Chamberlain, and Judith $S$. Eisen for commenting on this manuscript. Work on this project was supported by National Science Foundation Grant BNS8105746.

${ }^{2}$ Present address: Institute for Neuroscience, Department of Biology, University of Oregon, Eugene, OR 97403.

${ }^{3}$ To whom correspondence should be addressed.
}

light apparently does not vary in a circadian manner (Kaplan et al., 1980). Moreover, bath application of octopamine does not affect the excitation process significantly (Kaupp et al., 1983). While ventral photoreceptors do possess receptors for octopamine, as indicated by the octopamine-induced rise in cAMP (Kaupp et al., 1983), the physiological role of CAMP in the ventral eye is also unclear. Introduction of high concentrations of cAMP into ventral photoreceptors has only minor effects on excitation (Stern and Lisman, 1982). Similarly, excitation is not strongly affected by drugs that increase cAMP concentration (Corson et al., 1979; Brown et al., 1984).

We have re-examined the effect of octopamine and CAMP in the Limulus ventral eye, with particular attention to the process of darkadaptation, the process by which photoreceptors recover sensitivity after exposure to bright light. Our experiments indicate that octopamine and other drugs that increase cAMP concentration produce an increase in the rate of dark-adaptation. A brief report of this work has been presented (O'Day and Lisman, 1984).

\section{Materials and Methods}

Limulus polyphemus (horseshoe crabs) were obtained from the Marine Biological Laboratories, Woods Hole, MA in winter and spring. Ventral nerves were dissected free, desheathed, and superfused with artificial seawater (ASW) (Lisman and Brown, 1972). No attempt was made to control the time of the experiment or of sacrifice of the animal relative to the circadian rhythms of the animal. Individual photoreceptors were impaled with two microelectrodes and voltage-clamped to a holding potential of $-70 \mathrm{mV}$. In some experiments, cells were impaled with a single electrode to measure voltage. IBMX, theophylline, and octopamine were obtained from Sigma Chemical Co. Forskolin was obtained from Calbiochem-Behring Corp. Forskolin was solubilized in ethanol before addition to ASW (final ethanol concentration, $0.3 \%$ ). An equal concentration of ethanol was added to the control seawater in experiments involving forskolin and had only minor effects on responses.

The protocol for measuring photoreceptor dark-adaptation was as follows: To monitor the sensitivity of the photoreceptor to light, brief (10 msec), dim test flashes were presented at regular intervals (usually one per $5 \mathrm{sec}$ ). To light-adapt the photoreceptor, bright $\left(\sim 10^{-5} \mathrm{~W} / \mathrm{cm}^{2}\right)$ illumination from a second light source $(A D ;$; Fig. $1 A)$ was presented for several seconds. Test flashes were made considerably brighter during the adapting light in order to evoke measurable responses. When the adapting light was turned off, the test flash intensity was returned to its original level, and the cell gradually recovered sensitivity (dark-adapted). As the cell dark-adapted, responses to test flashes gradually increased, ultimately reaching a steady amplitude nearly equal to that observed before the adapting light. After a brief drug application, this entire protocol was repeated. By controlling the duration of the adapting light, the degree of desensitization produced by the light could be made almost exactly the same as that before drug application. Thus, the experiment was arranged so that responses to test flashes at the beginning and end of the dark-adaptation period were approximately the same in the control and tost situations. The expcrimental question was whether drug application would change the rate at which the cell made the transition between the light-adapted and dark-adapted states. In voltage-clamp experiments, lightserisitivity can be inferred directly from the amplitude of the current induced by the test flash, since the responses to brief dim flashes have been shown to vary approximately linearly with intensity under voltage-clamp conditions (Lisman and Brown, 1975a) 

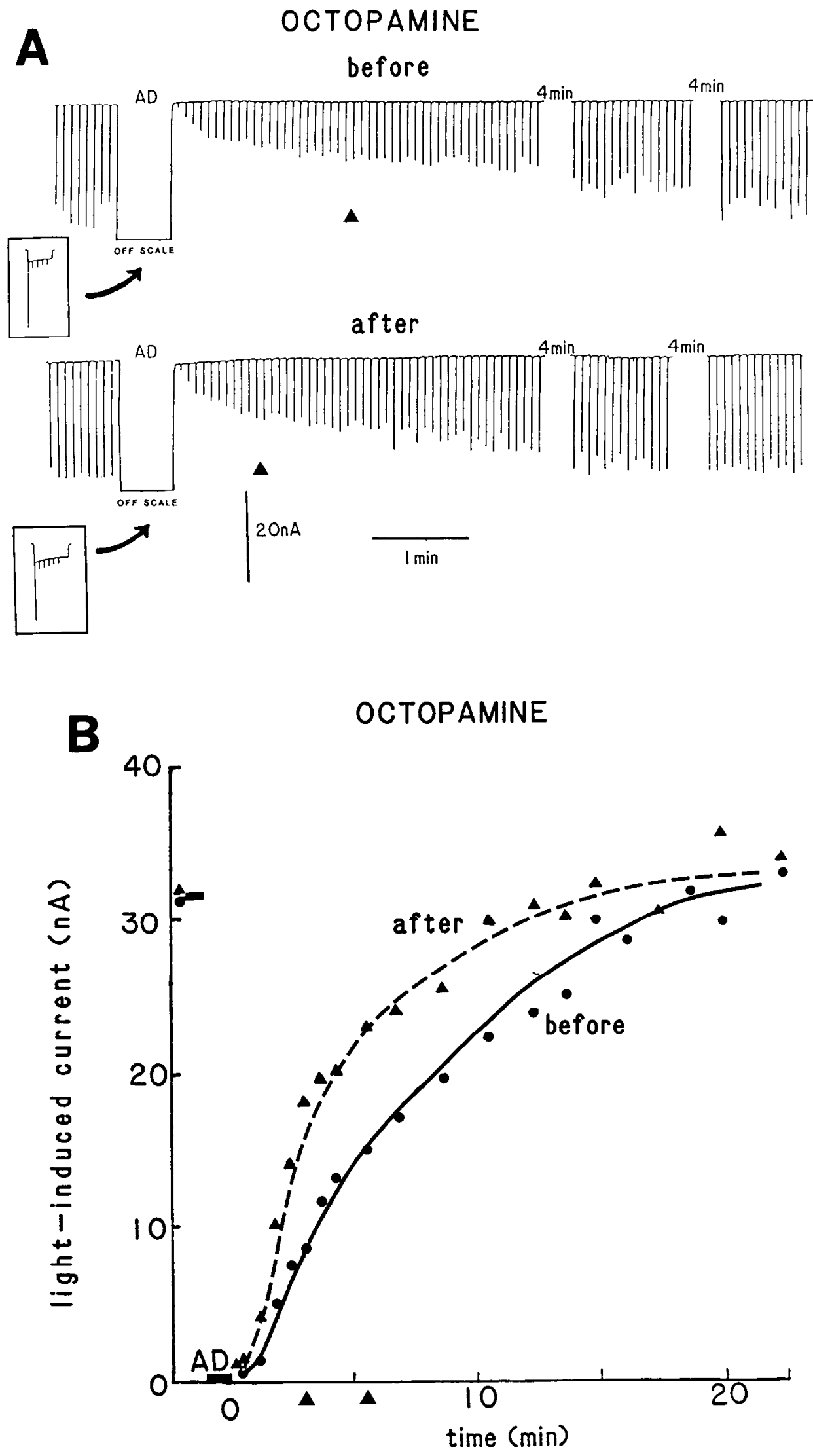

Figure 1. The rate of dark-adaptation was enhanced in voltage-clamped photoreceptors by octopamine application. $A, U p$ per trace: Test flashes evoked inward currents (downward deflections). Holding potential was $-70 \mathrm{mV}$. The adapting light $(A D)$ caused a desensitization from which the cell recovered to half-maximum in approximately 2 min (arrow) and completely in $13 \mathrm{~min}$. Lower trace: After application of a 4-min "pulse" of $100 \mu \mathrm{M}$ octopamine, the lightadapted photoreceptor recovered more rapidly. Recovery to half-maximum required approximately 1 min (arrow), and complete recovery required approximately $8 \mathrm{~min}$. This test run followed application of octopamine by $23 \mathrm{~min}$. $B$, Currents elicited by test flashes versus time after light-adaptation in a different photoreceptor than Figure $1 A$ Times to half-maximal recovery are indicated by arrows.

In some cells, octopamine caused slow changes in the absolute sensitivity (Fig. 4). Since we were uncertain of the relationship between dark-adaptation rates and the absolute sensitivity, we considered for further experimentation and analysis only cells in which the absolute sensitivity was not significantly changed by octopamine (see "Results").

\section{Results}

Octopamine increased dark-adaptation rates. The time course of dark-adaptation following an adapting light was monitored under voltage-clamp as described in "Materials and Methods." In the 


\section{OCTOPAMINE}
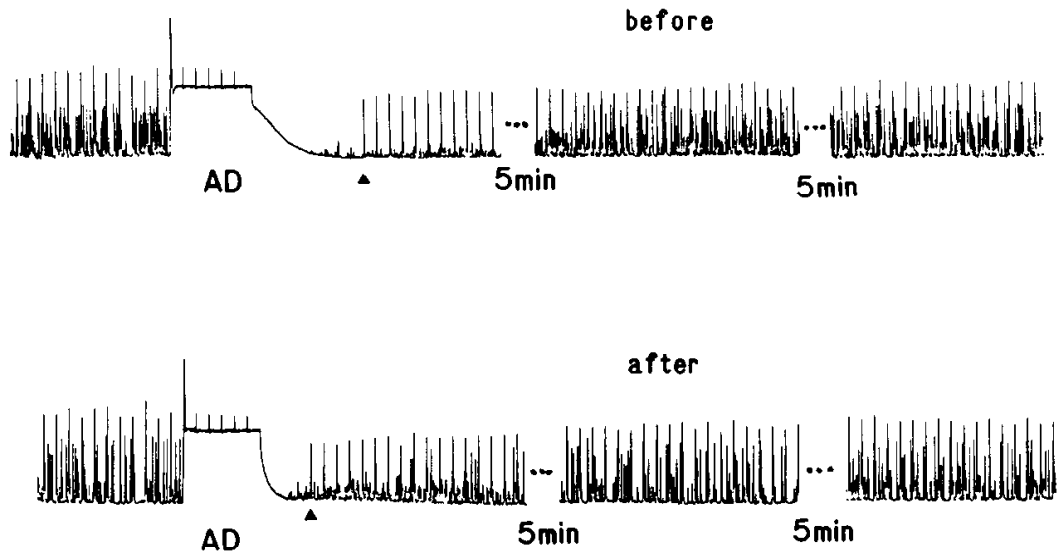

A

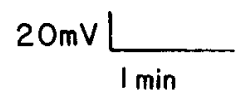

OCTOPAMINE

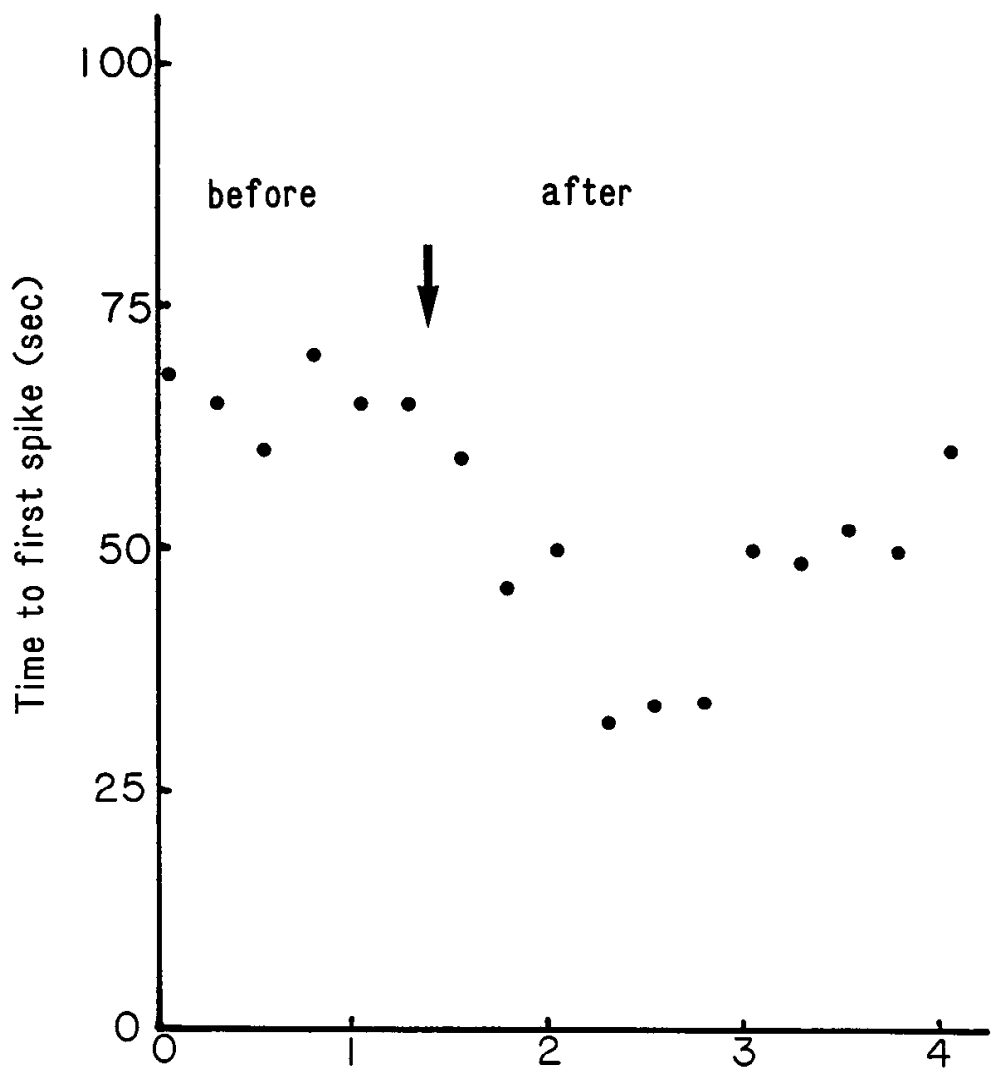

Figure 2. Octopamine enhances recovery of receptor potentials (current-clamp). $A$, The same protocol as in Figure 1 was used under current clamp. Resting potentials were -65 $\mathrm{mV}$ in both traces. Following a 5-min "pulse" of $100 \mu \mathrm{M}$ octopamine, the cell regained responsiveness following lightadaptation faster than before octopamine introduction. These traces were obtained 12 min after the "pulse" of octopamine. First spikes are indicated by arrows. The waveshape of the response to the adapting light was also changed by octopamine. Whether this was due to kinetic changes in the lightactivated conductance, to changes in voltage-dependent conductances, or to both remains unclear. $B$, The time to first spike following termination of the adapting light was measured as in $A$ for many successive cycles of light- and dark-adaptation. Time to first spike was initially stable; it decreased after application of octopamine and partially recovered within $2 \mathrm{hr}$. Adapting lights were given every $15 \mathrm{~min}$.
B

experiment illustrated in Figure $1 A$, the adapting light desensitized the photoreceptor in normal seawater by approximately 2.1 log units; after termination of the adapting light, the responses to test flashes recovered to half-maximum amplitude in $3.5 \mathrm{~min}$ and recovered completely in $13 \mathrm{~min}$. A brief ( 3 to $4 \mathrm{~min}$ ) "pulse" of octopamine (100 $\mu \mathrm{M})$ was then applied. Several minutes after the pulse, the photore- ceptor was again light-adapted by approximately 2.1 log units. Under these conditions, the recovery of the response after termination of the adapting light occurred more rapidly: to half-maximum in $1.2 \mathrm{~min}$ and completely in $8 \mathrm{~min}$. Similar results from another cell are shown in Figure $1 B$, which is a plot of the peak current responses to test flashes as a function of time after the light-adaptation, both before 

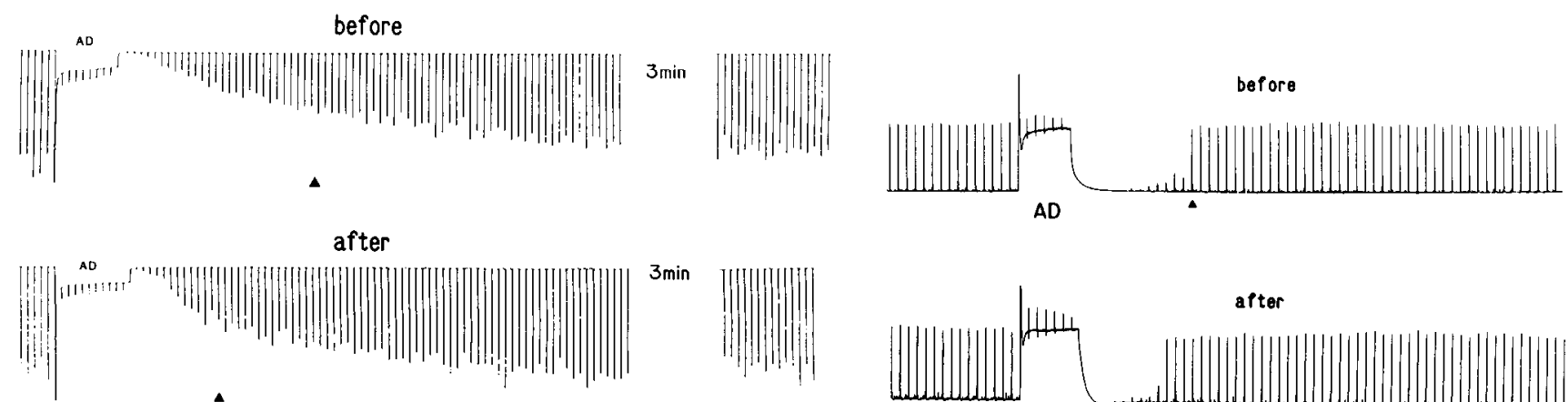

A
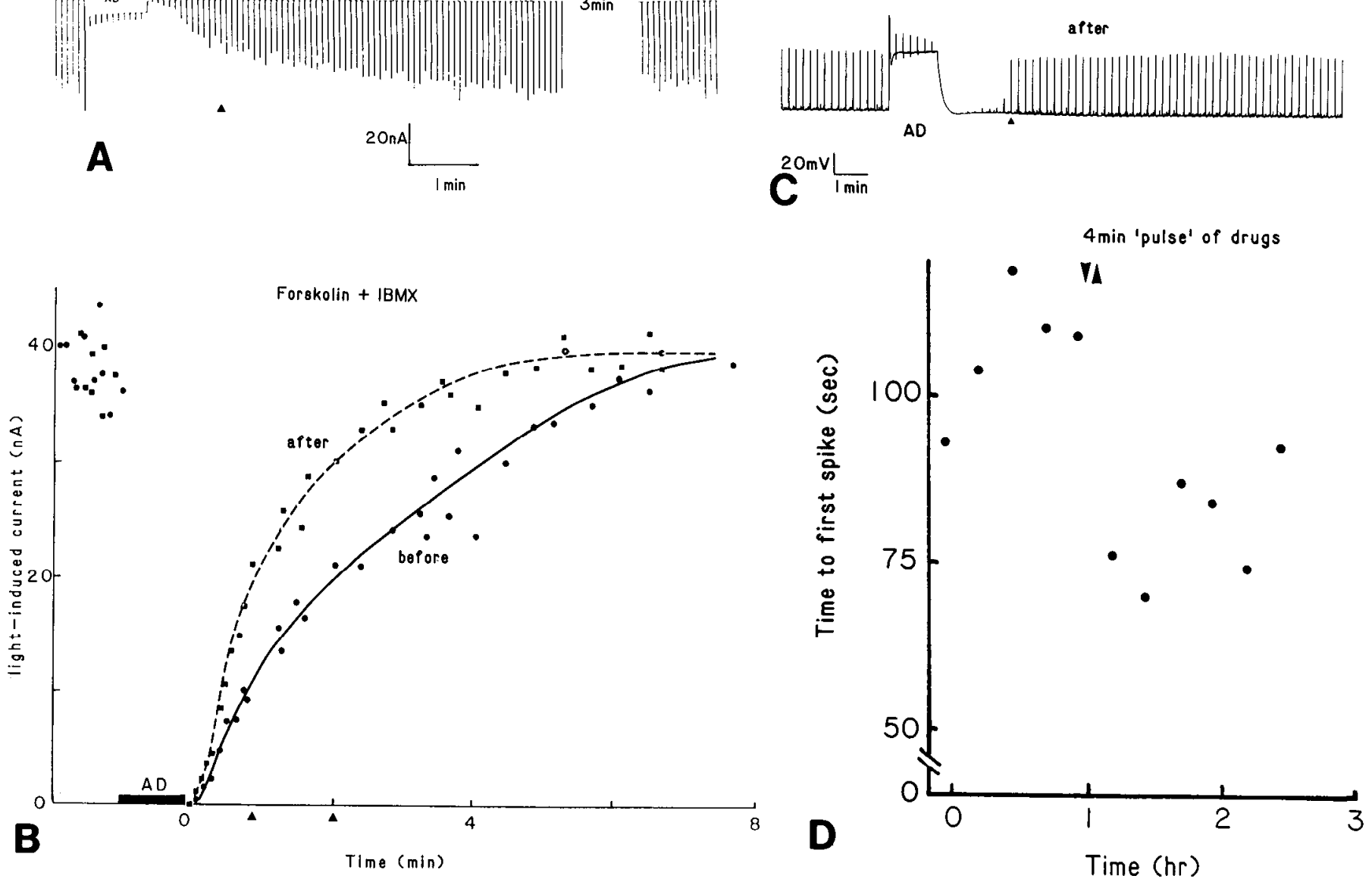

Figure 3. IBMX + forskolin enhance the rate of dark-adaptation. A, Forskolin (100 $\mu \mathrm{M})$ and IBMX (1 mM) were bath-applied to a photoreceptor voltageclamped to $-70 \mathrm{mV}$. The protocol was the same as that used in Figure 1. Before drug application, the recovery times following light-adaptation were approximately $2.5 \mathrm{~min}$ to half-maximal (arrow) and approximately $10 \mathrm{~min}$ to complete recovery. Following drug application, the recovery was faster. Halfmaximal recovery required approximately $1 \mathrm{~min}$, and complete recovery required approximately $5 \mathrm{~min}$. $B$, Peak currents evoked by test flash versus time from Figure 1A. C, IBMX (1 mM) and forskolin $(100 \mu \mathrm{M})$ enhanced the rate of recovery of receptor potentials. The protocol was the same as that used in Figure 2. Resting potential was $-67 \mathrm{mV}$ in the upper trace and $-68 \mathrm{mV}$ in the lower trace. $D$, Time to first spike following the adapting light versus time of the experiment for the same cell as in $C$.

and after a puise of octopamine. The enhanced rate of darkadaptation produced by octopamine persisted for at least an hour. At longer times, recordings sometimes became unstable or impalements could not be maintained and so, from these experiments, we are uncertain about how fast (or whether) the dark-adaptation rate returned to the control level. Octopamine enhanced dark-adaptation in six out of eight cells studied and had no effect in two cells. The effect on dark-adaptation was not readily apparent in experiments using dimmer adapting illumination.

A different method for studying the enhancement of dark-adaptation by octopamine is illustrated in Figure $2 A$. In experiments of this kind, the receptor potential was measured using a single microelectrode. This had the disadvantage of giving a less precise measure of cell sensitivity than the two-electrode voltage-clamp method used above, but it made possible longer experiments because of the improved recording stability. In these unclamped cells, a con- venient measure of dark-adaptation was the time required after the adapting stimulus for the appearance of the first light-induced regenerative spike (Millecchia and Mauro, 1969). This time indicates when the receptor potential had grown large enough to reach the spike threshold. To test the stability of the preparation, we light-adapted the cell and measured the subsequent dark-adaptation in several successive cycles before applying octopamine. A pulse of octopamine was applied only after four successive cycles in which the resting potential, dark sensitivity, and the rate of dark-adaptation were stable (Fig. $2 B$ ). Cells showed a negligible $(<2 \mathrm{mV}$ ) change in resting potential following the pulse of octopamine. In all cells studied in this way (three), the rate of dark adaptation was enhanced by octopamine. As illustrated in Figure $2 B$, there was a partial recovery of dark-adaptation rate $2 \mathrm{hr}$ after the octopamine pulse, but in no case was the recovery complete.

Forskolin and IBMX increased the rate of dark-adaptation. Kaupp 


\section{.}

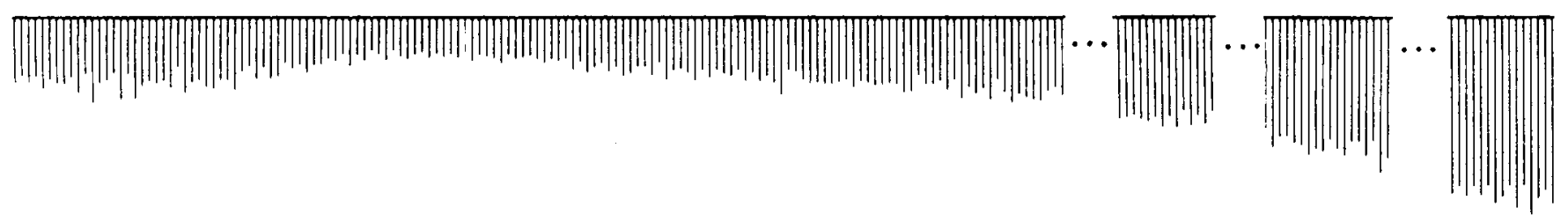

\section{$20 n A \underbrace{}_{1 \text { min }}$}

\section{$10 \min 25 \min 70 \mathrm{~min}$}

Figure 4. Agents which increase cAMP sometimes produced transient effects on sensitivity. Test flashes were presented at regular intervals to a voltageclamped cell (holding potential, $-70 \mathrm{mV}$ ). A 6-min "pulse" of $100 \mu \mathrm{M}$ forskolin and $1 \mathrm{mM}$ IBMX (given at arrow) produced a transient desensitization followed by a sensitization.

\section{OCTOPAMINE}

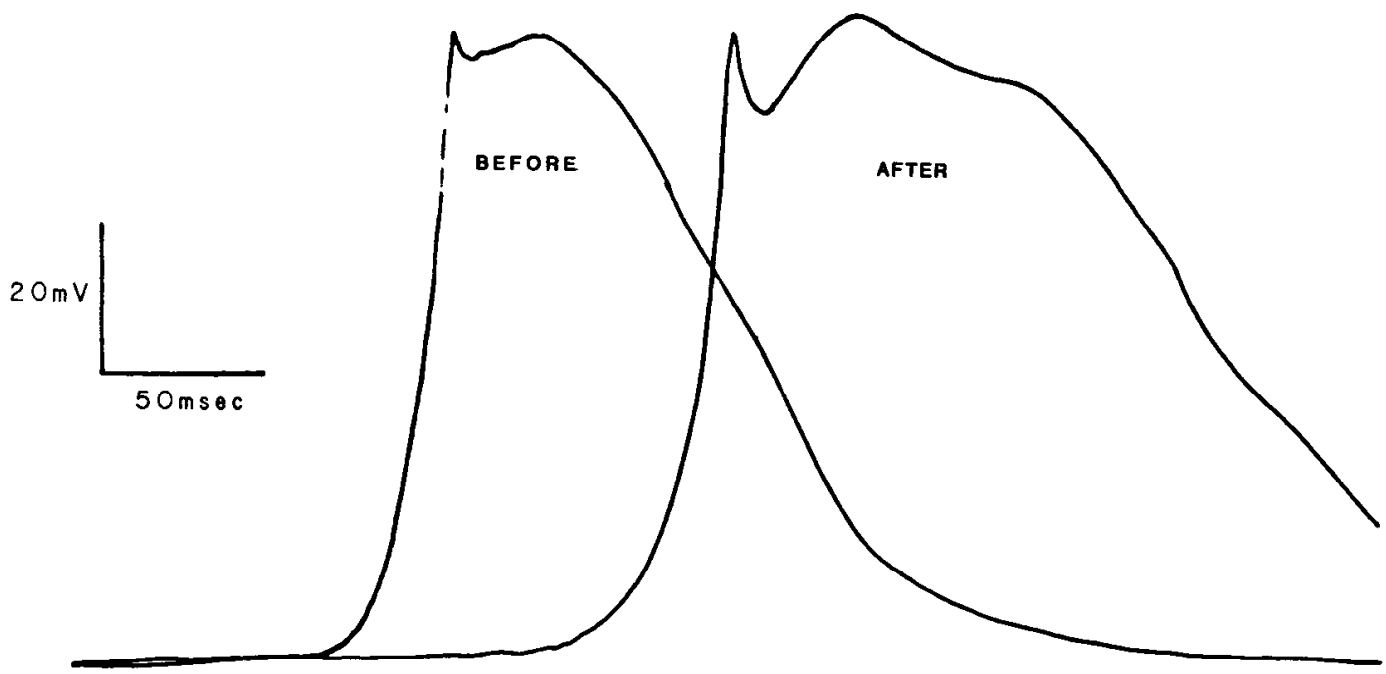

Figure 5. The kinetics of the receptor potential evoked by a $20-m s e c$ test flash was slowed by octopamine. Resting potential was $-68 \mathrm{mV}$ in both traces. The "octopamine" trace was recorded $30 \mathrm{~min}$ after a 5-min "pulse" of $100 \mu \mathrm{M}$ octopamine. $L \mathrm{M}$, light monitor.

et al. (1983) have shown that bath application of octopamine leads to an increase in the intracellular cAMP concentration in Limulus ventral photoreceptors. It therefore seemed possible that the action of octopamine on dark-adaptation rates was mediated by cAMP. To test this possibility, we introduced a combination of drugs shown previously to increase cAMP concentration dramatically (Brown et al., 1984): an adenylate cyclase activator, forskolin (100 $\mu \mathrm{M})$, and a phosphodiesterase inhibitor (either theophylline (1 $\mathrm{mM})$ or IBMX (1 $\mathrm{mm})$ ).

A pulse $(4 \mathrm{~min}$ ) of $100 \mu \mathrm{M}$ forskolin and $1 \mathrm{mM}$ IBMX (given simultaneously) produced a subsequent increase in the rate of darkadaptation in 10 of 10 cells studied. $100 \mu \mathrm{M}$ forskolin and $1 \mathrm{~mm}$ theophylline also increased the rate of dark-adaptation in two of two cells. Typical results are shown in Figure 3 . In this experiment, the photoreceptor was light-adapted by $2.4 \mathrm{log}$ units both before and after drug application. Before the drugs were introduced, the light sensitivity required $2.0 \mathrm{~min}$ to recover to half the pre-adaptation sensitivity and $8.5 \mathrm{~min}$ to complete recovery. After introduction of drugs, the recovery was faster: $1.0 \mathrm{~min}$ to half recovery and $5.0 \mathrm{~min}$ to complete recovery. We did not observe reversal of dark-adaptation rate, even after $2 \mathrm{hr}$ following removal of these drugs. Figure $3 D$ illustrates that these drugs also decreased the time-to-first spike, measured as in Figure 2.

After brief exposure to octopamine or to IBMX + forskolin, the dark-adapted sensitivity was usually fairly stable. Some cells, however, exhibited a transient decrease in sensitivity followed by a gradual and prolonged increase in sensitivity ( $\sim 0.3$ to $0.6 \log$ units) that was not reversible, at least for several hours. An experiment 
yielding such results is illustrated in Figure 4. Since the rate of dark adaptation might be linked somehow to the dark-adapted sensitivity, the comparison of dark-adaptation rates described in Figures 1 to 3 was restricted to cells in which drug application produced minimal changes in sensitivity ( $<0.1 \log$ unit; typically $0.06 \log$ units).

Kinetics of excitation were slowed after addition of CAMP-increasing agents. Direct introduction of CAMP into Limulus ventral photoreceptors by internal dialysis has been shown to increase the latency and slow the time course of the responses to test flashes (Stern and Lisman, 1982). Since octopamine increases the level of cAMP, one would expect octopamine to increase the latency and slow the kinetics. Figure 5 shows that this is the case. Addition of IBMX and forskolin also produced similar effects.

\section{Discussion}

We have found that a fundamental component of photoreceptor physiology, the dark-adaptation process, can be modulated by octopamine, the likely synaptic transmitter of efferent fibers that terminate on Limulus ventral photoreceptors (Battelle et al., 1982). Octopamine enhanced the dark-adaptation rate by as much as twofold, an enhancement that could be of considerable value to the organism. It remains, however, to be demonstrated that such modulation actually occurs during the natural circadian cycle of efferent input. Recently, Stieve and Andre (1983) have reported that octopamine increases the light sensitivity in unclamped ventral photoreceptors during light-adaptation, an effect that may be related to what we have found.

Octopamine acts in many cellular processes by activating an adenylate cyclase and increasing CAMP concentration (Nathanson and Greengard, 1973). In the ventral eye, Kaupp et al. (1983) demonstrated that octopamine potentiates adenylate cyclase activity and produces a rise in intracellular levels of cAMP. Thus, the effect of octopamine on dark-adaptation rates may be mediated by CAMP. This view is supported by evidence that pharmacological agents (IBMX and forskolin) that stimulate a rise in intracellular CAMP (Brown et al., 1984) produce an effect similar to that of octopamine. Similarly, in the lateral eye, application of a cAMP derivative mimics the effect of octopamine and of efferent activity (Kass and Barlow, 1982; Pelletier et al., 1984).

The fact that the dark-adaptation rate was still enhanced 1 to $2 \mathrm{hr}$ after a pulse of octopamine implies that recovery from the effects of octopamine must take more than $2 \mathrm{hr}$. Since Brown et al. (1984) found that the decline of CAMP following washout of forskolin and IBMX was relatively rapid (within $30 \mathrm{~min}$ ), it would follow that lowering cAMP does not by itself cause reversal. Thus, while dark-adaptation enhancement may require CAMP for initiation, the rate-limiting step in recovery may be due to a step subsequent to cAMP in the sensitivity control process. The time for reversal of adaptation rates is somewhat longer than would be expected on the basis of experiments in Limulus lateral eye where effects thought to be mediated by octopamine reverse completely in approximately 1 to $2 \mathrm{hr}$ (Barlow et al., 1977; Kaplan and Barlow, 1980). We have not examined the onset of the enhancement of dark-adaptation by drugs, but our evidence suggests that the effect occurs within 10 to $15 \mathrm{~min}$. This is consistent with the time course of the cAMP riso described by Brown et al. (1984)

Based on the key role of intracellular $\mathrm{Ca}^{2+}$ in light-adaptation in Limulus, it seems plausible that octopamine, possibly via CAMP, speeds dark-adaptation by enhancing the removal of $\mathrm{Ca}^{2+}$ from the cytoplasm. According to the Ca-hypothesis of adaptation in invertebrate photoreceptors (Lisman and Brown, 1972; Lisman and Brown, 1975b), the light-induced rise in intracellular $\mathrm{Ca}^{2+}$ (Brown and Blinks, 1974) is a key step in producing light-adaptation. It follows that the removal of intracellular $\mathrm{Ca}^{2+}$ is required for dark. adaptation. It is therefore attractive to imagine that both octopamine and the cAMP-increasing agents enhance dark-adaptation by stimulating a $\mathrm{Ca}^{2+}$ pump or $\mathrm{Na}$-Ca exchange mechanism responsible for removing $\mathrm{Ca}^{2+}$ from the cytoplasm (see review by Fain and Lisman,
1981). Consistent with this idea is the effect of the cAMP-increasing agents on the time course of individual responses to bricf flashes. The slowing of the response kinetics produced by octopamine cAMP-increasing drugs (Fig. 5), or cAMP (Stern and Lisman, 1982) resembles the slowing of response kinetics in low-Ca ${ }^{2+}$ seawater (Lisman, 1976). This effect of low extracellular $\mathrm{Ca}^{2+}$ is probably due to a reduction of intracellular $\mathrm{Ca}^{2+}$ concentration (Lisman, 1976). Octopamine might produce similar effects on kinetics by enhancing the $\mathrm{Ca}^{2+}$ pump and thereby lowering intracellular $\mathrm{Ca}^{2+}$. If this is the case, the response to octopamine in ventral photoreceptors would appear similar to the effect of $\beta$-adrenergic stimulation of vertebrate heart muscle, in which a rise in CAMP enhances $\mathrm{Ca}^{2+}$ pumping twofold, thereby leading to faster relaxation of the muscle (Tada and Katz, 1982; Lindemann et al., 1983).

\section{References}

Barlow, R. B., Jr. (1983) Circadian rhythms in the Limulus visual system. J Neurosci. 3: 856-870.

Barlow, R. B., Jr., S. J. Bolanowski, Jr., and M. L. Brachman (1977) Efferent optic nerve fibers mediate circadian rhythms in the Limulus eye. Science 197: 86-89

Barlow, R. P., Jr., S. C. Chamberlain, and J. Z. Levinson (1980) Limulus brain modulates the structure and function of the lateral eye. Science 210 . 1037-1039.

Ballelle, B.-A., J. A. Evans, and S. C. Chamberlain (1982) Efferent fibers to Limulus eyes synthesize and release octopamine. Science 216: 12501252.

Brown, J. E., and J. R. Blinks (1974) Changes in intracellular free calcium concentration during illumination of invertebrate photoreceptors: Detection with aequorin. J. Gen. Physiol. 64: 643-665.

Brown, J. E., U. B. Kaupp, and C. C. Malbon (1984) CAMP and adenylate cyclase in phototransduction by Limulus ventral photoreceptors. J. Physiol. 353: 523-539

Chamberlain, S. C., and R. B. Barlow, Jr. (1977) Morphological correlates of efferent circadian activity and light adaptation in the Limulus lateral eye. Biol. Bull. 153: 418-419.

Chamberlain, S. C., and G. A. Engbretson (1982) Neuropeptide immunoreactivity in Limulus. I. Substance P-like immunoreactivity in the lateral eye and protocerebrum. J. Comp. Neurol. 208: 304-315.

Corson, D. W. A. Fein, and J. Schmidt (1979) Two effects of phosphodiesterase inhibitors on Limulus ventral photoreceptors. Brain Res. 176: 365368.

Eisele, L. E., L. Kass, and R. B. Barlow (1982) Circadian clock generates efferent optic nerve activity in the excised Limulus brain. Biol. Bull. 163: 382.

Evans, J. A., S. C. Chamberlain, and B. Battelle (1983) Autoradiographic localization of newly synthesized octopamine to retinal efferents in the Limulus visual system. J. Comp. Neurol. 219: 369-383.

Fahrenbach, W. H. (1973) The morphology of the Limulus visual system. V. Protocerebral neurosecretion and ocular innervation. Z. Zellforsch. 144: 153-166.

Fain, G. L., and Lisman, J. E. (1981) Membrane conductances of photoreceptors. Prog. Biophys. Mol. Biol. 37: 91-147.

Kaplan, E., and R. B. Barlow, Jr. (1980) Circadian clock in Limulus brain increases response and decreases noise of retinal photoreceptors. Nature 286: 393-395

Kaplan, E., R. Batra, and R. B. Barlow, Jr. (1980) Recording from the Limulus ventral eye in situ. is there a circadian rhythm? Biol. Bull. 159: 486.

Kass, L., and R. B. Barlow, Jr. (1982) Efferent neurotransmission of circadian rhythms in Limulus lateral eye: Single cell studies. Biol. Bull. 163: 386.

Kass, L., and R. B. Barlow, Jr. (1984) Efferent neurotransmission of circadian rhythms in Limulus lateral eye. I. Octopamine-induced increases in retinal sensitivity. J. Neurosci. 4: 908.

Kaupp, U. B., C. C. Malbon, B.-A. Battelle, and J. E. Brown (1983) Octopamine stimulated rise of CAMP in Limulus ventral photoreceptors. Vision Res. 22: 1503-1506.

Lindemann, J. P., L. R. Jones, D. R. Hathaway, B. G. I lenry, and A. M. Watanabe (1983) Beta adrenergic stimulation of phospholamban phosphorylation and calcium ATPase activity in guinea-pig ventricles. J. Biol. Chem. 258: 464-471

Lisman, J. E. (1976) Effects of removing extracellular $\mathrm{Ca}^{2+}$ on excitation and adaptation in Limulus ventral photoreceptors. Biophys. J. 16: 1331-1335. Lisman, J. E., and J. E. Brown (1972) The effects of intracellular iontophoretic 
injection of calcium and sodium ions on the light response of Limulus ventral photoreceptors. J. Gen. Physiol. 59: 701-719.

Lisman, J. E., and J. E. Brown (1975a) Light-induced changes of sensitivity in Limulus ventral photoreceptors. J. Gen. Physiol. 66: 473-488.

Lisman, J. E., and J. E. Brown (1975b) Effects of intracellular injection of calcium buffers on light adaptation in Limulus ventral photoreceptors. J. Gen. Physiol. 66: 489-506.

Mancillas, J. R., and M. R. Brown (1984) Neuropeptide modulation of photosensitivity. I. Presence, distribution, and characterization of a substance P-like peptide in the lateral eye of Limulus. J. Neurosci. 4: 832846.

Millecchia, R., and A. Mauro (1969) The ventral photoreceptor cells in Limulus. II. The basic photoresponse. J. Gen. Physiol. 54: 310.

Natharisorl, A., and P. Greengard (1973) Octopamine sensitive adenylate cyclase. Evidence for the biological role of octopamine in nervous tissue. Science 180: 398-430.

O'Day, P. M., and J. E. Lisman (1984) Octopamine enhances dark-adaptation rates in Limulus ventral photoreceptors. Invest. Ophthalmol. Vis. Sci. 25: 235.

Pelletier, J. E., L. Kass, G. H. Rehninger, and R. B. Barlow, Jr. (1984) CAMP and octopamine partially mimic a circadian clock's effect on Limulus photoreceptors. Invest. Ophthalmol. Vis. Sci. 25: 288.

Stern, J. H., and J. E. Lisman (1982) Internal dialysis of Limulus ventral photoreceptors. Proc. Natl. Acad. Sci. U. S. A. 79: 7580-7584.

Stieve, H., and E. Andre (1984) Octopamine modulates the sensitivity of Limulus ventral photoreceptor. Z. Naturforsch. (C) 981-985.

Tada, M., and A. M. Katz (1982) Phosphorylation of the sacroplasmic reticulum and sarcolemma. Annu. Rev. Physiol. 44: 401-423. 